\title{
Is statistics meeting the needs of science?
}

\author{
Harry Crane* and Ryan Martin ${ }^{\dagger}$
}

January 10, 2018

\begin{abstract}
Publication of scientific research all but requires a supporting statistical analysis, anointing statisticians the de facto gatekeepers of modern scientific discovery. While the potential of statistics for providing scientific insights is undeniable, there is a crisis in the scientific community due to poor statistical practice. Unfortunately, widespread calls to action have not been effective, in part because of statisticians' tendency to make statistics appear simple. We argue that statistics can meet the needs of science only by empowering scientists to make sound judgments that account for both the nuances of the application and the inherent complexity of fundamental effective statistical practice. In particular, we emphasize a set of statistical principles that scientists can adapt to their ever-expanding scope of problems.
\end{abstract}

Keywords and phrases: Data science; p-hacking; reproducibility; scientific inference; validity.

\section{Introduction}

Today, average citizens are inundated by big data and quantitative analytics in their everyday consumption of politics, sports, and news coverage. Business decisions, government policies, and technical innovations are commonly guided by data driven solutions. And researchers in fields as diverse as anthropology and physics, psychology and botany, sociology and medicine are hard-pressed to publish their research without a supporting statistical analysis. These trends have elevated data science and statistics to the leading philosophy of modern times and have anointed statisticians as the de facto gatekeepers of scientific discovery. Well aware of the profound responsibility accompanying this newfound role, former President of the Institute of Mathematical Statistics (IMS), Richard Davis, titled his 2016 farewell address Are We Meeting the Challenge? (Davis 2016). He answers his own question with "an unequivocal yes!" But we're less convinced.

To be clear, our lack of conviction about whether statistics is meeting the challenge ought not be construed as skepticism about whether statistics can meet the challenge. We take the potential of statistical thinking in scientific inquiry to be self evident from the numerous scientific breakthroughs in which statistics has played an important role over

\footnotetext{
*Department of Statistics \& Biostatistics, Rutgers, the State University of New Jersey, hcrane@stat .rutgers.edu

${ }^{\dagger}$ Department of Statistics, North Carolina State University, rgmarti3@ncsu.edu
} 
the past century, as in the discoveries of a Higgs boson-like particle (Naik 2013) and of previously unknown forms of cancer (London Workshop 2013). Statistical thinking also plays a central role in emerging disciplines such as artificial intelligence and deep learning. These specific cases notwithstanding, our title question and the ensuing discussion reflects concern about whether statistics is living up to its vast potential for making the whole of science more rigorous, more efficient, and more effective. As we explain in the next section, overwhelming evidence suggests that statistics has not yet achieved this aim, and recent attempts by some statisticians to address certain issues are not heading in the right direction.

We, therefore, agree with many other prominent statisticians who have called attention to the troubling trends of statistical malpractice in scientific publication. But we believe the issues ought to be addressed differently. By now the problems are woven so deep into the fabric of scientific practice (e.g., Weisberg 2014) that they cannot be easily corrected by simply instructing practitioners to use one approach over another or to bear a few basic considerations in mind. Achieving far reaching impact requires a fundamental change to the mindset with which statistical analyses are approached. Towards this, we spell out a set of effectiveness principles to guide practitioners in their development of best practices for specific cases. Ultimately, the best method (statistical or otherwise) for a given scientific application ought to be determined by the scientists in charge of the investigation. By emphasizing the foundational thinking that underlies effective statistical practice in general, we propose here to empower practitioners with the ability to decide on their own which statistical approach, if any, is most suitable for addressing a particular question. We hope that this approach will also help to solidify and clarify statisticians' role in the "data science" movement.

\section{$2 \quad$ A troubling trend}

Rising right alongside the use of statistical methods and data driven solutions in science and industry is the rate at which scientific journal articles are being retracted (Steen et al 2013). The role of flawed statistical analyses in these retractions is widespread and has been well understood since at least a decade ago, when Ioannidis (2005) produced a poignant mathematical argument for "Why most published research findings are false." But despite increased public outcry, there remain serious concerns about the effects of statistical malpractice, e.g., the widespread inability to reproduce statistically significant findings (Nature 2017) and the related p-hacking crisis (Gelman and Loken 2014; Nuzzo 2014), on both science and society more broadly.

In $p$-hacking, statistical models, methods, or hypotheses are chosen not to address a particular scientific question but rather to obtain a statistically significant $p$-value. The problem is widespread and obviously troublesome, and yet it persists throughout scientific publications of the highest caliber. The persistent problem has caused some to respond in dramatic fashion. The journal Basic and Applied Social Psychology (BASP) has banned the use of $p$-values in its published articles (Trafimow and Marks 2015), to which the American Statistical Association (ASA) responded with an official statement on $p$-values (Wasserstein and Lazar 2016). The unprecedented nature of the BASP ban and ensuing ASA statement underscores the pervasiveness of the problem. They also 
demonstrate that while scientists and statisticians agree that there is a problem, they have a different understanding of its root cause and how it should be addressed. The situation is so dire, in fact, that a horde of 72 prominent statisticians have recently called to "redefine statistical significance" (Benjamin et al 2017) by changing the conventional $p$-value threshold from 0.05 to 0.005 . The proposal has since faced mounting criticism for its over-simplification of a complex problem and overly optimistic projection of its consequences (Amrhein and Greenland 2017; Crane 2017; Lakens et al 2017; McShane et al 2017; Perezgonzalez and Frias-Navarro 2017; Trafimow et al 2017).

Though the scope of our discussion extends beyond the debate concerning $p$-values, part of our message can be understood by comparing to the $B A S P p$-value ban. The $p$ value ban is certainly extreme, seeking to eliminate $p$-hacking by eliminating $p$-values and any other methods related to null hypothesis significance testing. And while we do not advocate any bans or mandates of particular methods, we recognize the potential value of the $B A S P$ ban in that it at least forces scientists to rethink their approach. Statisticians, on the other hand, are reluctant to place the blame for bad science on the $p$-values: the problem lies with the misapplication of $p$-values, not with the $p$-values themselves. But this reluctance to take the necessary steps to improve the state of affairs has resulted only in bureaucratic initiatives, like the ASA statement, which ultimately expend a lot of effort to accomplish very little. As Wasserstein and Lazar (2016) themselves admit in their introduction to the ASA statement:

Nothing in the ASA statement is new. Statisticians and others have been sounding the alarm about these matters for decades, to little avail.

Indeed, if nothing in the statement is new, then how could it be expected to have any noticeable impact?

While the ASA statement has been met by some criticism (e.g., Ionides et al 2017), it largely reinforces the attitude, common in statistics circles, that statisticians aren't to blame for the current mess. It's true that $p$-hacking and other more run-of-the-mill statistical missteps are now so commonplace that no one community is to blame. But the "do no harm" attitude reflected in the ASA statement and other contemporary memes, such as "Being a statistician means never having to say you're certain" (e.g., Gandy Jr. 2001), do not exonerate statisticians of culpability. Statisticians may not have created the problem, but we are now all too aware of its severe consequences to allow the status quo to perpetuate. Statisticians are uniquely qualified to reverse the trend so that statistics can deliver on its promise to produce more rigorous and more effective science.

\section{Meeting the needs of science}

Rather than asking whether statistics is meeting the needs of science, some statisticallyminded readers may prefer instead to ask: Is scientific practice living up to the standards of statistics? The evidence presented in Section 2 suggests that the answer to this latter question is No. This is a key takeaway of not only the ASA statement but also the "how to" guides published recently by other prominent statisticians (Kass et al 2016; Yu 2016) as well as in other discussions taking place regularly on statistics-themed blogs ${ }^{1}$ and at

\footnotetext{
${ }^{1}$ e.g., http://andrewgelman.com and https://simplystatistics.org
} 
statistics conferences. $^{2}$ It is of little use to repeat this message here.

The answer to our title question-Is statistics meeting the needs of science? - is less clear, as can be seen in the divergence between the above discussed trends and the message of Davis's farewell address to the IMS. It is apparent, through the widespread incorporation of statistical training in undergraduate and graduate scientific curricula as well as the across-the-board trend for scientific articles to be supported by statistical analysis, that scientists recognize the value of statistical thinking. Yet the statistical community, through the ASA statement and other less formal means, continue to sound the alarm about inappropriate uses of statistics throughout the sciences.

Taken together, these points suggest that scientists very much want to live up to statistical standards, but they have been unable to. While there are many possible explanations for this shortcoming, the jumbled message about statistical principles conveyed by the statistical community is surely a contributing factor. Though many statisticians take these principles seriously, others just roll their eyes. Case in point, Speed (2016) writes

If a statistical analysis is clearly shown to be effective [...] it gains nothing from being described as principled.

Though a statistician might be careful in parsing Speed's words - he doesn't explicitly denounce statistical principles, but rather dismisses principles as superfluous to statistical practice - most non-statisticians are likely to take Speed's message at face value. Speed's emphasis on effectiveness over principle presents statistics as a tool instead of as a way of thinking. Effectiveness is a worthy goal of any practical endeavor, but what makes a statistical method effective? Speed doesn't say. For sure, a judgment about a method's effectiveness is steeped in deeply ingrained statistical principles which, though perhaps obvious to Speed and other statisticians, aren't obvious to scientists. Such dismissals of principles by statisticians of Speed's stature undermine the role of statistics in science by trivializing the depth of knowledge and keen instincts needed to effectively carry out a statistical analysis.

Given what's at stake, statisticians need to be honest about the complexity of the systems under study and the amount of skill required to analyze them properly. Achieving effectiveness is not as simple as Speed's remark above or the recent "how to" guides written by Kass et al (2016) and Yu (2016) suggest. The discussion in Kass et al (2016), for example, gives "Ten simple rules" for effective statistical practice, among which are listed such generic aphorisms as "Plan Ahead" and "Keep it Simple." Though presented in an oversimplified manner, a careful reader with the appropriate training (i.e., a statistician) can readily tease out their subtle points. But readers with such expertise have no need for these guidelines in the first place. Instead, in presenting these simple rules in the way that they do, Kass et al give the impression that achieving effective statistical practice is, well, simple.

Several decades ago, Barnard (1985), extended the opposite message to statisticians:

We need more complexity; and this should win us greater recognition from scientists in developed areas, who already appreciate that inference is a complex

\footnotetext{
${ }^{2}$ e.g., https://ww2 . amstat.org/meetings/ssi/2017/index.cfm
} 
business while, at the same time, it should deter [others] from thinking that all they need is a suite of computer programs.

The point of Barnard's statement is not that complexity should be added to statistical analysis; it's that the complexity is already there. Statisticians ought to acknowledge that statistical practice is complex, relying on nuanced principles honed through years of experience. Carrying out an effective analysis is far from simple, and suggestions that it can or even ought to be simple serve to both mislead scientists as well as diminish the role of statisticians in sound scientific practice.

As the well-known saying goes, "Give a man a fish and feed him for a day. Teach a man to fish and feed him for a lifetime." Societal trends toward efficiency tend to promote pre-packaged statistical software, generalist approaches to undergraduate education, and the simplified message found in the various "how to" guides cited above. These measures satisfy the scientist's statistical needs for only a day. As the use of statistics becomes more widespread and the problems to which statistical thinking are applied continue to evolve, the statistical methods best suited to meeting today's scientific needs quickly become obsolete. The effectiveness principles below are intended to serve as the basis of effective statistical analysis that can satisfy the scientist's needs for much longer.

\section{Effectiveness principles}

Any meaningful statistical analysis is guided by a question, and the effectiveness of such an analysis is to be evaluated based on how useful it is for addressing that question. With this in mind, we stress here that statistical analyses are not right or wrong but rather effective or ineffective for addressing a well defined scientific question. Two very different approaches may nevertheless be equally effective for addressing the same problem. And just as a statistical analysis cannot be right or wrong, neither can the data, the model, or the inference. As we articulate below, data is relevant or irrelevant (to the given question), the model is sound or unsound (for the given data), and the inference is valid or invalid (for the given model). Taken together, a statistical analysis is effective or ineffective (for answering the given scientific question), and, à la Abelson (1995), it is the data analyst's job to present an argument that these effectiveness principles have been met.

\subsection{The data must be relevant}

To perform a meaningful statistical analysis, one must first have a question. In classical statistical applications, such as clinical trials, agricultural field studies, and the like, this question is articulated well before statisticians arrive on scene: a pharmaceutical company wants to test whether a new drug treats diabetes better than the current standard; a farmer wishes to determine which fertilizer-soil combination provides the best crop yield; a biologist explores how certain diseases are genetically linked, as in the now-classical gene expression studies (Golub et al 1999; Hedenfalk et al 2001) and more recent genome-wide association studies (Klein et al 2005; Wellcome Trust Case Control Consortium 2007; Visscher et al 2012). The data for addressing such questions may be collected through a carefully designed experiment, by an observational study, by combing through a large database, or possibly some other means. Regardless, in all of these cases, the data 
has been implicitly deemed to be relevant to the scientific problem because it has been collected with that problem in mind.

In more modern applications, the data may be collected from a hodgepodge of complex, uncontrollable sources, in real time and in enormous quantities. Examples abound in the technological age, in which massive-scale data collection efforts, such as those by Google (2017) and the National Security Agency (NSA, 2017), are commonplace. Unlike the setting of the preceding paragraph, such 'Big Data' problems are rarely motivated by a concrete scientific question at the time the data is being collected. For example, the NSA-operated Intelligence Community Comprehensive National Cybersecurity Initiative Data Center, or Utah Data Center for short, stores all data about U.S. citizen website visits, Internet searches, phone calls, etc. in a single massive facility. The data is collected for the general purpose of national security but without any pre-existing security concern in mind. The rationale here is that when a security concern arises, the data stored in the Utah Data Center will be relevant to addressing it.

Both classical and modern cases highlight that, regardless of how the data is collected or where it originates, to be useful the data's relevance to the scientific question or practical problem must be adequately articulated. The data stored in the Utah Data Center is not immediately relevant to any given national security problem; it only becomes relevant once, say, a suspected security threat is identified and the nature of the threat is analyzed vis-à-vis the information stored in the database.

Articulating data relevance is just the first step in an effective statistical analysis, as it serves only to establish a connection between observations and the system under investigation. The determination of data relevance most often involves extra-statistical expertise, such as intuition and knowledge gained from the existing scientific theory. In many cases, the data is obviously relevant to the question at hand, just as drug trial outcomes are relevant to drug efficacy, crop yield is relevant to fertilizer productivity, and gene expression is relevant to genetic profiles. The statistical insight lies not in determining that the data is relevant, but rather in how the data is relevant. How the data is relevant figures crucially into model construction and subsequent inferences.

\subsection{The model must be sound}

Whether or not statistical analyses are employed, relevant data is a minimal requirement of any scientific investigation. In articulating how the data is relevant, the investigator identifies certain sources of variability and uncertainty for which the analysis ought to account. For inferences to be meaningful, they ought to generalize beyond the single study on which they are based and, therefore, the model should provide an adequate description of the sources of variation in the observed data as well as in any other data that could have been obtained under the same scientific conditions. A sound model meets both of these objectives.

Establishing model soundness depends on the role played by the model in a given application. Two typical roles of statistical models - there can be others-are:

- To establish the relationship between observed data and the hypothetical population from which data has been sampled; or

- To describe the mechanism by which the data has been generated. 
Both involve extrapolation beyond what is observed, either through the sampling mechanism or the generating process. It is in establishing a formal extrapolation mechanism that the model factors most prominently in statistical inference; see Section 4.3. In either case, the soundness of the model is determined by how closely the conceptual mechanism for extrapolation mirrors the actual relationship between observed and unobserved data.

Predictive analyses leading up to the 2016 U.S. Presidential election offer a poignant example about the importance of accounting for the relationship between population and sample. Although the polls mostly pointed to a Clinton victory over Trump, the analyses of these polls by Nate Silver's FiveThirtyEight website, Sam Wang's Princeton Election Consortium, and many others largely failed to account for uncertainty and biases in how the polling was conducted (Gelman 2016), leading to analyses which overstated Clinton's advantage. These sampling imperfections prompted Wang to assert that "the polls were off" (Lohr and Singer 2016) when, in fact, the models were off precisely because the manner in which the polling data was "representative" of the population voting behavior was not properly incorporated into the analysis.

Our emphasis on model soundness, instead of model correctness, may remind some of that old saw "All models are wrong but some are useful" (Box and Draper 1987). The principle of model soundness, however, should eliminate this cliché from the statistical vernacular. Quite simply, models are not right or wrong, but rather sound or unsound, as judged relative to how well they capture uncertainty and promote sensible inferences from the data. A sound model provides the necessary link between the data and the inferences that ultimately guide scientific conclusions. For any given application, a lot of specific considerations go into identifying a sound statistical model. For example, when assessing soundness of a model for sampled or generated data, one may compare certain observed statistics with their known behaviors under the posited model, akin to residual analysis in ordinary regression.

A more specific related to model soundness is the maxim by Cox and Hinkley (1974, p. 5): There should be consistency with known limiting behavior. So, if a generative model produces a data sequence whose limiting behavior differs substantially from known empirical behavior, the model must be unsound. For example, the pursuit of consistency with observed limiting behavior motivated Crane and Dempsey's (2017) development of edge exchangeable network models, which can capture the sparsity patterns in observed social and biological networks without compromising subsequent inferences. For the sake of brevity, we do not discuss the many other subtle issues which figure into specific applications. We only note that the assessment of model soundness is a major challenge in modern data science and "big data" problems in general, and deserves careful attention in any statistical analysis.

\subsection{The inference must be valid}

Often the goal of a statistical analysis is to make inferences about the system under investigation. We emphasize here that inference is a conclusion based on evidence and reasoning, not a hypothesis test, effect size, etc. In our context, evidence is the output of a statistical analysis, along with any other relevant information, and reasoning is a subjective, but logical, judgment. Given that inference is subjective, drawing conclusions is actually relatively easy; anyone can do it, in any way they'd like. The challenge lies 
in drawing conclusions that are scientifically justifiable, or valid. Clearly, a justification cannot be based on the derived conclusion being scientifically correct, since this truth is never available to the data analyst, at least not at the time of the analysis. A more realistic goal is to avoid "systematically misleading conclusions" (Reid and Cox 2015) that can be detrimental to the advancement of science. As we describe below, this requires a connection between the data analyst's subjective judgments and the extrapolation mechanism described by the model.

Towards this, let $D$ denote relevant data and $\mathrm{P}_{\theta}$ a sound model, indexed by a parameter $\theta \in \Theta$. The scientific problem suggests a hypothesis $A \subset \Theta$ about the system being studied. Rarely is it possible to prove that $A$ is true or false based on $D$ alone, but it is possible to make certain subjective judgments about the plausibility of $A$ based on the data and other relevant information. Towards this, the investigator defines a function $\mathrm{pl}_{D}(\cdot)$ for which $\mathrm{pl}_{D}(A)$ measures the plausibility of hypothesis $A$ based on data $D$. (The mathematical details are beyond our present scope but see our comments below about the comparison to $p$-values.) Based on this function, the investigator can draw certain subjective conclusions, e.g., by specifying a cutoff $c$ and concluding that $A$ is sufficiently implausible if $\mathrm{pl}_{D}(A) \leq c$.

The investigator's subjective judgment attains scientific meaning by connecting the plausibility function, on which the judgment is based, to the sound model, which describes the system to which the conclusions apply. In other words, scientific meaningfulness requires that the investigator's plausibility function satisfy certain model-specific conditions. In particular, following the logic in Fisher (1973, Ch. 3), the event where a true hypothesis is judged to be sufficiently implausible must have low probability under the model. More precisely, we say that the investigator's inference about the hypothesis $A$, based on $\mathrm{pl}_{D}(\cdot)$, is valid, at a (small) specified level $\alpha \in(0,1)$, if

$$
\mathrm{P}_{\theta}\left\{\mathrm{pl}_{D}(A) \leq c\right\} \leq \alpha, \quad \text { for all } \theta \in A \text {. }
$$

In words, the probability of judging $A$ to be sufficiently implausible (i.e., $\operatorname{pl}_{D}(A) \leq c$ ) must be no greater than the specified $\alpha$ under any candidate distribution $\mathrm{P}_{\theta}$. If condition (1) is satisfied, then the inferences based on $\mathrm{pl}_{D}$ are valid in that the method by which they are drawn avoids systematically misleading conclusions (Martin and Liu 2016).

Though $p$-values are a special case of the more general class of plausibility functions satisfying (1) - see Martin and Liu (2014) and Martin (2017) for details - we emphasize that $\mathrm{pl}_{D}$ is not merely a $p$-value in disguise. Moreover, there are advantages to a plausibility-centric framework. First, interpretation of the derived conclusions is more straightforward, which should help avoid the confusion that surrounds significance testing. That is, rejecting a null hypothesis is not a discovery, but rather is a judgment that the hypothesis is sufficiently implausible based on data and any other relevant information. Second, there is a precise mathematical framework for plausibility functions that is arguably more appropriate for scientific reasoning compared to probability. For example, plausibility functions are not additive, so $\mathrm{pl}_{D}(A)$ being small does not imply $\mathrm{pl}_{D}\left(A^{c}\right)$ is big, consistent with the understanding that additional evidence in direct support of $A^{c}$ is required to justify a discovery. These points align with scientists' common sense, but they are subtle and, therefore, not simple. Unfortunately, the standard presentation of statistical inference has been streamlined so much over the years, for the sake of making the process simple, that this fundamental understanding has been lost. 


\section{Conclusion}

In raising the title question, Is statistics meeting the needs of science?, we have highlighted the divergence between statistical thinking, as perceived by statisticians, and statistical practice, as implemented by non-statistician scientists. To reiterate our point from earlier, we have no doubt that statistics can meet the needs of science, but we also feel strongly, supported in large part by the ongoing $p$-hacking and reproducibility crises, that statistics is not currently meeting those needs. We have argued here that realizing the great potential of statistics for scientific discovery requires that we first recognize the need for a firm grounding in the principles of effective statistical practice. As statisticians, it is then our responsibility to impart these principles in a clear and insightful way so that scientists can put them to best use in their own substantive domains. To this end, we have identified the three essential components of effective statistical practice - relevant data, sound model, and valid inference - and outlined a strategy for communicating these essentials to scientists. In keeping with Barnard's advice, our discussion here is an attempt to reverse the "simplicity" trend cultivated by many high-profile statisticians with an emphasis on the core principles for effective statistical practice and that living up to these principles is far from simple. Beyond highlighting these principles, we have refrained from outlining specific guidelines for how to implement these principles in practice. At least one root cause of the issues we have discussed here is the paradigmatic view that statistical procedures ought to follow a fixed, formulaic protocol, e.g., the orthodox implementation of null hypothesis significance testing in most areas of science. This conventional viewpoint has obvious and well-documented drawbacks, which $B A S P$ has sought to mitigate with its $p$-value ban. We hope to improve on these efforts by emphasizing statistics as a principled mindset and way of thinking, not just a simple tool.

Our discussion about principles vs. practice goes hand-in-hand with the "value vs. visibility paradox" (Gibson 2017), according to which the value of statistical thinking is widely recognized but statisticians are mostly invisible from the practical implementation of those ideas. This lack of visibility in the modern practice of data science has led to the concern among many statisticians that we are missing the boat on the data science phenomenon. It is perhaps with this concern in mind that Marie Davidian, then president of the ASA, posed the question "Aren't we data science?" (Davidian 2013). The numerous published replies to Davidian ranging the yes/no/maybe spectrum reflect a growing identity crisis in the statistical community. While statistical thinking is and always will be a vital part of data science, the disconnect between statistical foundations and their practical implementation has left statisticians wondering what role, if any, they play in data science. We ask: Have statisticians distinguished themselves from the computer scientists, mathematicians, and others who also lay claim to data science?

It is with this precise question in mind that the ASA issued a statement ${ }^{3}$ On the Role of Statistics in Data Science which describes the benefits of "a statistical framework" and "statistical methods" in the context of data science. A stark omission from this earlier ASA statement is any mention of statistical principles or statistical insights, the skills that draw most heavily on the instincts and abilities of statisticians. If the ASA can't even identify what statisticians bring to the table, is it any wonder that researchers in other areas have replaced statisticians in the data science conversation? When viewed

\footnotetext{
${ }^{3}$ http://ww2 . amstat.org/misc/DataScienceStatement.pdf
} 
in this light, it comes as no surprise that statisticians are in danger of "missing the boat," precisely because they have habitually diminished their standing among their interdisciplinary peers. Rather than retread the common misinterpretations of $p$-values, the ASA should have issued a comment on the core principles of statistical practice, establishing the value of statisticians and statistical thinking in the wide range of modern scientific problems to which it is now applied. Since they did not, we were happy to help meet this need.

\section{References}

Abelson, R. (1995). Statistics as Principled Argument. Psychology Press.

Barnard, G. A. (1985). A coherent view of statistical inference. Technical report, Department of Statistics and Actuarial Science, University of Waterloo.

Benjamin, D.J., et al. (2017). Redefine statistical significance. Nature Human Behaviour.

Crane, H. (2017). "Redefining Statistical Significance" will not 'immediately improve' reproducibility and could make the replication crisis worse. Preprint available at https://psyarxiv.com/bp2z4/.

Crane, H. and Dempsey, W. (2017). Edge exchangeable models for network data. J. Amer. Statist. Assoc., to appear; preprint available at arXiv:1603.04571.

Cox, D. R. and Hinkley, D. V. (1974). Theoretical Statistics. Chapman and Hall, London.

Davidian, M. (2013). Aren't we data science? AmStat News, July, p. 3-5.

Davis, R. A. (2016). Presidential Address: "Are we meeting the challenge?" IMS Bulletin, October, p. 1,4-7.

Dempster, A. P. (1989). Logicist statistics. I. Models and modeling. Statist. Sci., 13(3), $248-276$.

Fisher, R. A. (1973). Statistical Methods and Scientific Inference, Haffner Press, New York.

Gandy Jr., O. H. (2001). Journalists and academics and the delivery of race statistics: being a statistician means never having to say you're certain. Race and Society, 4, 149-160.

Gelman, A. (2016). Survey weighting and that $2 \%$ swing. http://andrewgelman.com/ 2016/12/01/survey-weighting-2-swing/. December 1, 2016.

Gelman, A. and Loken, E. (2014). Data-dependent analysis - a "garden of forking paths" - explains why many statistically significant comparisons don't hold up. Am. Sci., 102(6):460.

Gibson, E. W. (2017). Leadership in statistics: Increasing our value and visibility. Am. Stat., to appear. 
Golub, T. R. et al (1999). Molecular classification of cancer: Class discovery and class prediction by gene expression monitoring. Science, 286, 531-537.

Google (2017). Privacy policy, https://www.google.com/policies/privacy/. August 21, 2017.

Hedenfalk, I. et al (2001). Gene-expression profiles in hereditary breast cancer. N. Engl. J. Med., 344, 539-548.

Ioannidis, J. P. A. (2005). Why most published research findings are false. PLOS Med 2(8): e124.

Ionides, E. L., Giessing, A., Ritov, Y., and Page, S. E. (2017). Response to the ASA's statement on p-values: context, process, and purpose. Am. Stat., 71, 88-89.

Kass, R. E. et al (2016). Ten simple rules for effective statistical practice. PLOS Comput. Biol., 12:e1004961.

Klein, R. J. et al (2005). Complement factor H polymorphism in age-related macular degeneration. Science, 308, 385-389.

Lakens, D., et al. Justify Your Alpha: A Response to "Redefine Statistical Significance". Preprint available at https://psyarxiv.com/9s3y6.

Lohr, S. and Singer, N. (2016). How data failed us in calling an election. The New York Times. http://www.nytimes.com/2016/11/10/technology/the-data-said-clintonwould-win-why-you-shouldnt-have-believed-it.html. November 10, 2016.

London Workshop (2013). Statistics and Science: A Report of the London Workshop on the Future of the Statistical Sciences. http://www.worldofstatistics.org/ wos/pdfs/Statistics\&Science-TheLondonWorkshopReport.pdf

Martin, R. (2017). A mathematical characterization of confidence as valid belief. Preprint available at https://arxiv.org/abs/1707.00486.

Martin, R. and Liu, C. (2014). A note on p-values interpreted as plausibility. Statist. Sinica, 24, 1703-1716.

Martin, R. and Liu, C. (2016). Validity and the foundations of statistical inference. Preprint available at https://arxiv.org/abs/1607.05051.

McCullagh, P. (2002). What is a statistical model? Ann. Statist., 30(5):12251310. With comments and a rejoinder by the author.

McShane, B. B., Gal, D., Gelman, A., Robert, C., and Tackett, J. L. (2017). Abandon statistical significance. Preprint available at https://arxiv.org/abs/1709. 07588.

Naik, G. (2013). New Data Boosts Case for Higgs Boson Find. The Wall Street Journal. March 14, 2013.

National Security Agency (2017). Domestic Surveillance Techniques, https://nsa. gov1.info/surveillance/, August 21, 2017. 
Nature (2017). Challenges in Irreproducible Research, Nature News \& Comment. http: //www.nature.com/news/reproducibility-1.17552. Retrieved on August 21, 2017.

Nuzzo, R. (2014). Scientific method: Statistical errors. Nature, 506:150-152.

Perezgonzalez, J. D. and Frias-Navarro, D. (2017). Retract 0.005 and propose using JASP, instead. Preprint available at https://psyarxiv.com/t2fn8.

Reid, N. and Cox, D. R. (2015). On some principles of statistical inference. Int. Statist. Rev., 83(2):293-308.

Speed, T. (2016). Terence's Stuff: Principles. IMS Bulletin, September, p. 17.

Steen, R. G., Casadevall, A., and Fang, F. C. (2013). Why has the number of scientific retractions increased? PLOS ONE 8(7): e68397.

Trafimow, D. et al (2017). Manipulating the alpha level cannot cure significance testing - comments on "Redefine statistical significance". PeerJ Preprints.

Trafimow, D. and Marks, M. (2015). Editorial. Basic Appl. Soc. Psych., 37(1):1-2.

Visscher, P.M., Brown, M.A., McCarthy, M.I., Yang, J. (2012). Five years of GWAS discovery. Am. J. Hum. Genet., 90(1): 7-24.

Wasserstein, R. L. and Lazar, N. A. (2016). The ASA's statement on p-values: context, process, and purpose. Am. Stat., 70:129-133.

Weisberg, H. I. (2014). Willful Ignorance: The Mismeasure of Uncertainty. John Wiley \& Sons, Inc., Hoboken, NJ.

Wellcome Trust Case Control Consortium (2007). Genome-wide association study of 14,000 cases of seven common diseases and 3,000 shared controls. Nature, 447(7145): 661678 .

Yu, B. (2016). Data wisdom for data science. IMS Bulletin, September, p. 8-9. 